\title{
EVALUATION OF MANDATORY CASE-BASED REPORTING SYSTEM FOR VARICELLA IN THE PREVACCINE ERA
}

\author{
Maja Sočan \\ Centre for Communicable Diseases, National Institute of Public Health, Ljubljana, Slovenia
}

\begin{abstract}
SUMMARY
Varicella is a mandatory reportable disease in Slovenia but it is not notifiable in most European countries. The aim of the study was to explore the completeness of mandatory varicella reporting in Slovenia. Data were extracted from three electronic datasets, including: mandatory notifiable communicable diseases dataset (SURVIVAL), health statistics dataset - consultations in primary care (ZUBSTAT), and health statistics dataset - hospitalizations (BOLOB). Age- and sex-specific reporting rates were compared during the period 1997-2006. During the ten-year period studied 168,089 cases were recorded via ZUBSTAT, and 128,222 cases by SURVIVAL. On average $76.2 \%$ (range: 62.9\%-94.9\%) were notified. In both datasets, the notified incidence of varicella was highest for preschool children, with a downward shitt in age at contracting varicella. The percentage of notified cases decreased with increasing age. The number of hospitalized cases was low for BOLOB and SURVIVAL. On average $74 \%$ of hospitalized cases were reported. The mandatory notification system in Slovenia provides enough information to survey age/sex-specific varicella trends in the prevaccine era.
\end{abstract}

Key words: varicella, surveillance systems, notification, reporting, completeness, electronic dataset

Address for correspondence: M. Sočan, Centre for Communicable Diseases, National Institute of Public Health, Trubarjeva 2, 1000 Ljubljana, Slovenia. E-mail: maja.socan@ivz-rs.si

\section{INTRODUCTION}

Epidemiological data on communicable diseases constitute a basic component of the risk assessment and management, and of the evaluation of prevention programmes (1). The data are collected through routine mandatory case-based and laboratory reporting systems, sentinel surveillance systems and, occasionally, through cross-sectional studies. Data on vaccine preventable diseases are mostly obtained from seroprevalence studies (2).

Mandatory reporting systems are crucial for continuous and timely surveillance of communicable diseases. Reporting systems are usually enforced by law which obligates the physician or/and laboratory to notify communicable diseases (3). Previous studies, however, showed that practicing physicians declined to report (4-9). The main reasons for underreporting are physicians' unfamiliarity with laws, and their lack of motivation and knowledge about the importance of good quality surveillance data $(10,11)$.

The reporting completeness was studied for several communicable diseases. There are very few studies addressing varicella notification systems (4-6). In most countries, varicella is not a notifiable disease $(12,13)$. According to the existing Communicable Diseases Act, varicella is one of notifiable communicable diseases in Slovenia (14). Data on notified varicella cases are currently collected and aggregated, and sex/age specific disease trends are analyzed yearly. The collected data are not used to trigger public health action.

Varicella vaccine, which was developed in the seventies, will be included in the Slovenia vaccination programme for preschool children, most probably as a combined measles-mumps-rubellavaricella vaccine, and data collected through the case-based surveillance system will be used to evaluate the vaccination programme. The reliability of the obligatory notification system for varicella will become even more important.

The aim of the present study was to evaluate the obligatory notification system for varicella used in Slovenia in the prevaccine era by comparing the existing surveillance data against the data derived from health statistics datasets for a ten-year period.

\section{METHODS}

\section{Data Sources}

Data were derived from the following three electronic datasets: mandatory notification system, and health statistics datasets for consultations in primary care and for hospitalizations.

\section{Mandatory Notification System}

Patients with uncomplicated varicella are seldom seen more than once. They usually do not consult their doctor on the day they are taken ill, but rather on the second or third day, or even later. After the consultation, a notification form is filled in. It includes the following data: name, surname, date of birth, permanent address, time of onset of varicella symptoms/signs, notification date, hospitalization, outcome data and information on whether or not the diagnosis was confirmed by microbiological tests. The 
diagnosis of varicella was invariably clinical - microbiological tests (isolation/PCR of varicella-zoster virus or serology) were done only exceptionally.

The filled in notification forms were sent to regional institutes of public health to be entered into an electronic dataset (SURVIVAL). The updated electronic files were forwarded to the National Institute of Public Health on a weekly basis.

\section{ZUBSTAT Health Statistics Data Set - Consultations in Primary Care}

Outpatient primary health care physicians (in public or private practice under concession) coded each first visit for illness according to the Slovenia version of the International Classification of Diseases 10th Revision (ICD-10), and nurses entered the code into an electronic dataset ZUBSTAT - Outpatient Statistics. Primary health care institutions send aggregated depersonalized datasets to regional institutes of public health twice a year (latest by 20 July for the first half of the year, and latest by 20 January for the second half). Regional public health institutes checked the data received for completeness and accuracy, and transmitted the corrected data to the national-level institution.

Basic demographic information (sex- and age-segregated data in 14 age groups) is obtained using a special programme created for primary health care statistics. Other data (e.g. day/month of visit, disease visit rate, therapy, duration of sick leave) useful for assessment of the disease burden are not collected routinely.

ZUBSTAT electronic dataset contains only aggregated data and no personal data. It was therefore not possible to identify patients entered into both datasets i.e. health statistics and mandatory notification system.

\section{BOLOB Health Statistics Dataset - Hospitaliza- tions}

Public and private hospitals under concession are obligated to report hospitalization data directly to the National Institute of Public Health twice a year (by 25 July for first half-year and by 15 February for second half-year). This reporting system was introduced in 1997 and was harmonized as recommended by the WHO and EU. Diseases, injuries and intoxications are coded according to ICD-10.

For each hospitalized patient his/her personal identification data (coded with personal registration number), sex, length of hospital stay, outcome, a principal and secondary diagnosis were entered into the BOLOB electronic dataset. At birth every Slovenia citizen is registered with the Central Population Register and assigned a unique personal registration number.

Both, SURVIVAL electronic dataset for mandatory notifiable communicable diseases and BOLOB electronic hospitalisation dataset contain personal data. SURVIVAL comprises the patient's name and surname (not personal registration number), and BOLOB the patient's personal registration number without name and surname, which disables a direct comparison of patients in both datasets.

Data retrieved from all three electronic datasets included the number of cases coded as varicella (B01), varicella meningitis (B01.0), varicela encephalitis (B01.1), varicella pneumonitis (B01.2), varicella with other complications (B01.8), varicella without complications (B0.9) betweeen 1997 and 2006. The varicella cases were stratified by age (age groups : $<1,1-3,4-6$, $7-9,10-14,15-19,>20$ ) and sex, and trends in age distribution of varicella cases were calculated.

\section{RESULTS}

During a ten-year period (1997-2006), 128,222 varicella cases, $(63,953-49.9 \%$ males and $64.269-50.1 \%$ females) were notified in Slovenia. The number of cases varied from 9,178 in 2005 (incidence: 458.9 per 100,000 population) to a maximum of 15,728 cases in 1998 (incidence: 786.4 per 100,000 population), the average annual number being 12,822 cases (Table 1 ).

During the first five years (1997-2001), the notified varicella rate was highest in 4-6-year-olds, and during the next five-year period, in 1-3-year-olds (Fig. 1). A decreasing trend was observed for all age groups of four years and above. During the same period, 168,089 first visits to out-patients clinics for 85,867 (51.1\%) males and for 82,222 (48.9\%) females were coded as B.01 varicella with or without complications (Table 1 ). The rate of varicella cases recorded in ZUBSTAT health statistics dataset was highest for children 1 to 6 years of age (Fig. 2). Age-specific distribution of varicella cases showed a downward trend for all age groups, but was seen to increase for children younger than 4 years. The comparison of both datasets showed that the percentage of cases notified through national surveillance system varied from $62.9 \%$ to $94.9 \%$. The overall notification rate was $76.2 \%$ (Table 1 ).

Table 1. Varicella consultations (Health Statistics Dataset) and notified varicella cases for a ten-year period

\begin{tabular}{|l|c|c|c|c|}
\hline Year & $\begin{array}{c}\text { Health } \\
\text { statistics } \\
\text { dataset } \\
\text { - ZUBSTAT }\end{array}$ & $\begin{array}{c}\text { Incidence } \\
\text { rate - } \\
\text { ZUBSTAT } \\
\text { (per 100,000) }\end{array}$ & $\begin{array}{c}\text { Notified } \\
\text { cases } \\
\text { (percentage } \\
\text { of all cases) }\end{array}$ & $\begin{array}{c}\text { Incidence } \\
\text { rate - notified } \\
\text { cases } \\
\text { (per 100,000) }\end{array}$ \\
\hline 1997 & 16,525 & 826.2 & $\begin{array}{c}13,919 \\
(84.2 \%)\end{array}$ & 695.9 \\
\hline 1998 & 20,643 & $1,032.1$ & $\begin{array}{c}15,728 \\
(76.2 \%)\end{array}$ & 786.4 \\
\hline 1999 & 20,834 & $1,041.7$ & $\begin{array}{c}14,242 \\
(68.4 \%)\end{array}$ & 712.1 \\
\hline 2000 & 17,555 & 877.7 & $\begin{array}{c}12,877 \\
(73.4 \%)\end{array}$ & 643.8 \\
\hline 2001 & 11,651 & 582.5 & $\begin{array}{c}11,065 \\
(94.9 \%)\end{array}$ & 553.2 \\
\hline 2002 & 16,084 & 804.2 & $\begin{array}{c}12,137 \\
(75.5 \%)\end{array}$ & 606.8 \\
\hline 2003 & 18,033 & 901.6 & $\begin{array}{c}15,294 \\
(84.8 \%)\end{array}$ & 764.7 \\
\hline 2004 & 16,401 & 820.0 & $\begin{array}{c}12,928 \\
(78.8 \%)\end{array}$ & 646.4 \\
\hline 2005 & 14,586 & 729.3 & $\begin{array}{c}9,178 \\
(62.9 \%)\end{array}$ & 458.9 \\
\hline 2006 & 15,777 & 788.8 & $\begin{array}{c}10,854 \\
(68.8 \%)\end{array}$ & 542.7 \\
\hline Total & 168,089 & 840.4 & $\begin{array}{c}128,222 \\
(76.2 \%)\end{array}$ & 641.1 \\
\hline
\end{tabular}




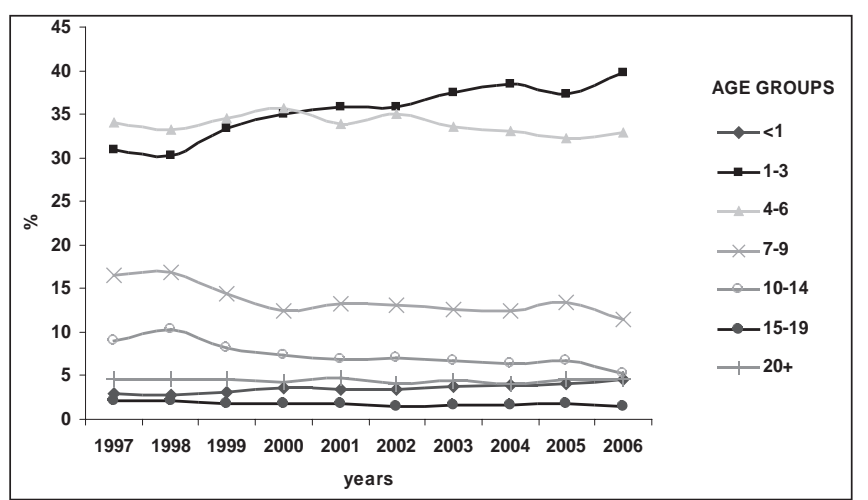

Fig. 1. Percentage of notified varicella cases (SURVIVAL), by age, 1997-2007.

Table 2. Hospitalisation data for notified varicella cases and cases reported via the health statistics system

\begin{tabular}{|l|c|c|}
\hline Year & $\begin{array}{c}\text { Health information } \\
\text { system } \\
\text { - hospitalizations }\end{array}$ & $\begin{array}{c}\text { Hospitalized notified } \\
\text { varicella cases (percentage } \\
\text { of all hospitalized patients) }\end{array}$ \\
\hline 1997 & 90 & $84(93 \%)$ \\
\hline 1998 & 95 & $70(74 \%)$ \\
\hline 1999 & 97 & $75(77 \%)$ \\
\hline 2000 & 110 & $62(56 \%)$ \\
\hline 2001 & 100 & $73(73 \%)$ \\
\hline 2002 & 123 & $81(66 \%)$ \\
\hline 2003 & 133 & $112(84 \%)$ \\
\hline 2004 & 101 & $61(60 \%)$ \\
\hline 2005 & 76 & $66(87 \%)$ \\
\hline 2006 & 97 & $75(77 \%)$ \\
\hline Total & 1022 & $759(74 \%)$ \\
\hline
\end{tabular}

Provided that the total number of varicella cases seen by doctors equalled the number of first visits to primary care clinics, the notified incidence rate was highest in children under one year of age, followed by toddlers (more than $80 \%$ of all cases). The notification rate in young adults was less than $50 \%$ (Fig. 3).

Few varicella patients needed hospitalisation. According to the health statistics hospitalization dataset (BOLOB), 1,022 (0.61\%) of all varicella patients were admitted to acute care hospitals during the ten-year period studied. Practically the same percentage was recorded through the mandatory surveillance system: 759 (0.59\%) of the 128,222 notified varicella cases needed hospital care.

The number of hospitalized cases can serve as a proxy indicator of the number of severe cases of the disease. The comparison of data derived from health statistics and of data provided by the notifiable disease surveillance system showed that the notification rate was not higher for severe cases, and that on average $74 \%$ of hospitalized cases were reported (Table 2).

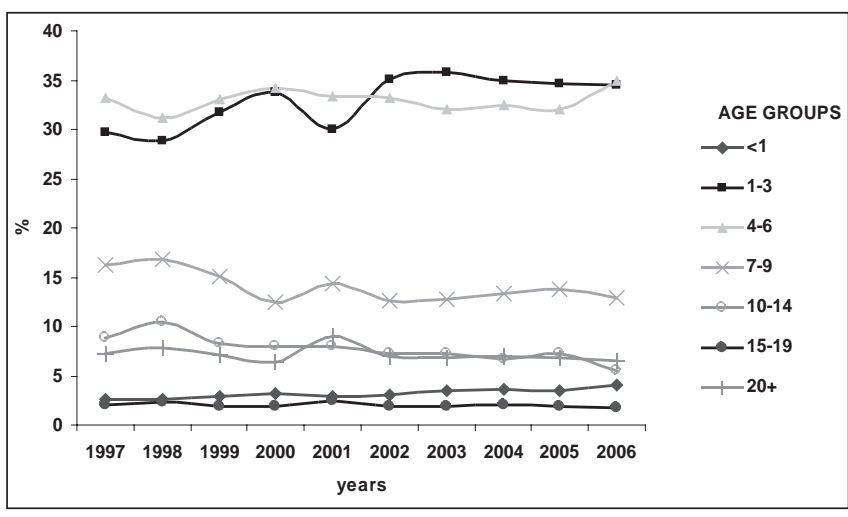

Fig. 2. Percentage of varicella cases, by age, 1997-2007 (health statistics electronic dataset ZUBSTAT).

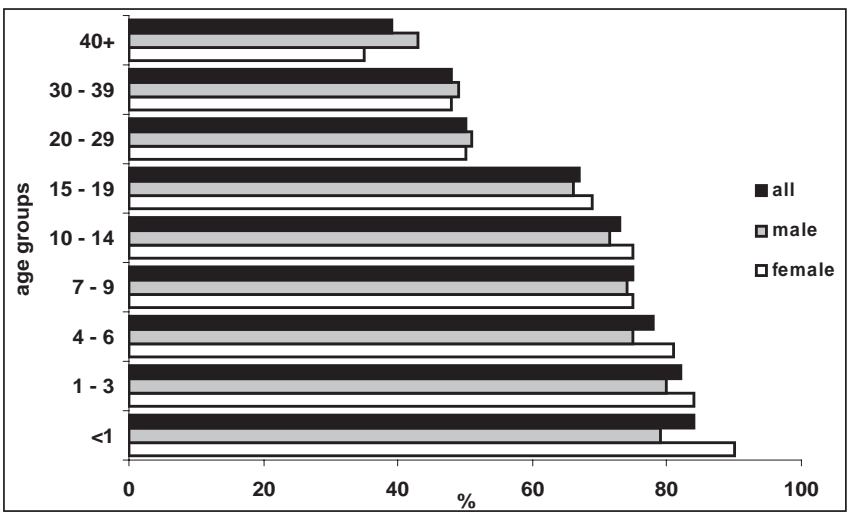

Fig. 3. Percentage of notified cases, by age groups, during a ten-year period.

\section{DISCUSSION}

Our study indicates that during a ten-year period three quarters of varicella patients seen by a physician were reported through the national notifiable disease surveillance system.

Varicella is one of the commonest vaccine-preventable childhood diseases in countries with temperate climate. The development of varicella vaccine dates back more than thirty years. Nevertheless, only few EU countries have introduced or are considering introducing universal childhood varicella vaccination (12). The United States incorporated varicella vaccination into the routine immunization schedule in 1995. A massive vaccination programme in the United States resulted in a reasonably rapid fall in the incidence of varicella in immunized children, and exhibited a cohort effect on the non-immunized part of the population. The surveillance data showed that a single dose of vaccine given at approximately 15 months of age did not provide the expected level of protection (15). Breakthrough infections and outbreaks in schools and kindergartens have been described (16). In response to this recently recognized problem a two-dose 
schedule has been introduced. In the United States, varicella was added to the national notifiable disease list in 2003 (17). The purpose of case-based surveillance programmes was to monitor closely the changing epidemiological pattern of varicella, and to identify vaccine failures.

Among the EU member states, Cyprus, Italy, Latvia, Malta, Slovakia, Slovenia and Ireland (for outbreaks only) have casebased surveillance programmes. Some of these countries collect aggregate data on varicella, and others, e.g. France, have established a primary health care-based sentinel surveillance system (12).

The Communicable Diseases Act of 1977 added varicella to the list of obligatorily notifiable diseases in Slovenia. Personal data and some epidemiological data are collected via a casebased surveillance system. It was found that a surprisingly high number of varicella patients seen by primary care physicians were notified. There are few studies evaluating the completeness of reporting communicable diseases; the situation is especially unfavorable for varicella, which is not a notifiable communicable disease in the majority of countries. A Taiwanese study compared data provided by the notifiable disease surveillance system with data on annual outpatient health insurance claims during a threeyear period (6). A very low (6.45\%) and inconsistent reporting rate was found for the notifiable diseases system which should provide data to estimate the disease prevalence and vaccine efficacy. The reporting rate was highest in children under one year of age. In Slovenia, a much higher reporting rate was found for age groups cared for by paediatricians or school physicians than for those attending general practitioners. In Slovenia, the majority of preschool children are seen by paediatricians. Primary and secondary school students visit school physicians (specialists in school medicine) who provide services for a given number of schools in the community. Paediatricians and school physicians carry out periodic preventive health examinations and vaccination of preschool and school children, and offer curative care to their patients. During their training, paediatricians and school physicians receive more information about the importance of timeliness and completeness of their reports than do general practitioners, and they are therefore more willing to report. Because the consultation rate for infectious diseases is much higher in children than in adults, they see more outbreaks of communicable viral respiratory and gastrointestinal diseases than their colleagues in general practice. Consequently, they more often collaborate with regional epidemiologists. Regional epidemiologists are vaccination programme coordinators, and they daily communicate with physicians performing vaccinations to discuss unconventional vaccine schedules, unexpected side effects and sometimes to solve problems with inadequate vaccine procurement. We assume that a more intense communication between this segment of primary care and communicable diseases epidemiology influences the notification rate for infectious diseases in general, as observed in one of the previous studies (18).

Seroprevalence studies demonstrated varicella specific antibodies in more than $90 \%$ of ten-year-olds (19). During the prevaccine period, the majority of varicella cases were in children and primary school students seen by paediatricians and school physicians. If these doctors continue to report in the future, the traditional passive surveillance system relying on the clinician's initiative will provide a reasonable quantity of data needed to follow trends in age- and sex-specific varicella rates. On the basis of the data collected we concluded that the longest reporting delay between disease onset and date of reporting was more than month. In practice, the time interval between a visit to the doctor and data gathering at the national level is no more than a fortnight (data not shown).

Some countries developed sentinel surveillance systems for a limited number of communicable diseases $(20,21)$. These systems have higher specificity and better timeliness then mandatory surveillance systems, but they are less sensitive for detecting outbreaks of less common diseases (22). Paediatric sentinel surveillance of vaccine-preventable diseases in Italy showed that the national estimates are 3 to 7 times higher than those obtained through statutory notifications (5). According to Moretti, who compared the sentinel surveillance system to the obligatory notification system, the underreporting rate for varicella was $43 \%$. He concluded that voluntary participation of physicians in the sentinel network provides good quality data, making these networks very useful tools for the epidemiological evaluation of infectious diseases (4). In Slovenia, the present notification system provides adequate and timely information on varicella in the prevaccine era. We believe that there is no need to introduce a sentinel surveillance system for varicella.

Two drawbacks are apparent in the notification system used: it requires a lot of paper work for the reporting physician. It adds an extra burden on a busy outpatient clinic and constitutes a barrier to efficient and rational reporting. There is a need to introduce electronic reporting of communicable disease surveillance data. The positive impact of electronic clinical and laboratory reporting systems has been demonstrated in many countries in terms of improved timeliness and completeness. Increased reporting efficiency leads to higher public health awareness of communicable diseases (23-25).

The implementation of an electronic reporting system would overcome another drawback of the present system, i.e. agedependent differences in reporting. The reporting rate is found to decrease with increasing age. After the initiation of varicella vaccine immunization, the case-based surveillance of varicella will be particularly important in the group with (possible) lower level of immunity. The current surveillance system for varicella provides adequate information to follow the disease dynamics and detect outbreaks. Yet, its credibility may become questionable with a lower overall rate of varicella and an increased burden of the disease among adolescents and adults.

In conclusion, assessment of the completeness of the present obligatory notification system in Slovenia showed that the data collected are consistent and representative over time. After the introduction of varicella vaccination, improved notification system will be needed to enhance surveillance in all age groups, especially in adolescents and adults. Closer collaboration with practicing physicians and epidemiologists will raise awareness of the importance of a reliable notification system, and stress the need for the implementation of electronic reporting system.

\section{Acklowledgements}

The study was supported by the Slovenian Research Agency, Research Programme [grant number: P3-0339], Research in Public Health. The author would like to thank M. Blaško,V. Prodan, and A. Zgaga for the technical assistance in data input and to T. Kustec for trend analysis. 


\section{REFERENCES}

1. Amato-Gauci A, Ammon A. The surveillance of communicable diseases in the European Union - long-term strategy (2008-2013). Euro Surveill. 2008 Jun 26;13(26):pii 18912.

2. German RR, Lee LM, Horan JM, Milstein RL, Pertowski CA, Waller MN; Guidelines Working Group Centers for Disease Control and Prevention (CDC). Updated guidelines for evaluating public health surveillance systems: recommendations from the Guidelines Working Group. MMWR Recomm Rep. 2001 Jul 27;50(RR-13):1-35.

3. Commission Decision of 22 December 1999 on the communicable diseases to be progressively covered by the Community network under Decision No 2119/98/EC of the European Parliament and of the Council (notified under document number C(1999) 4015). 2000/96/EC. Off J Eur Communit. 2000 Feb 3;42(L28):50-3.

4. Moretti F, Chellini E, Baretti S, Santini MG, Rosati GV, Comodo N. Estimate of underreporting of infectious diseases through a sentinel network of pediatricinas in the area of local health unit of Florence. Epidemiol Prev. 2000 Sep-Oct;24(5):224-7. (In Italian.)

5. Ciofi Degli Atti ML, Salmaso S, Bella A, Arigliani R, Gangemi M, Chiamenti G, et al; Pediatric Sentinel Surveillance Study Group. Pediatric sentinel surveillance of vaccine-preventable diseases in Italy. Pediatr Infect Dis J. 2002 Aug;21(8):763-8.

6. Tan HF, Chang CK, Tseng HF, Lin W. Evaluation of the National Notifiable Disease Surveillance System in Taiwan: an example of varicella reporting. Vaccine. 2007 Mar 30;25(14):2630-3.

7. Brabazon ED, O`Farrell A, Murray CA, Carton MW, Finnegan P. Under-reporting of notifiable infectious disease hospitalisations in a health board region in Ireland: room for improvement? Epidemiol Infect. 2008 Feb;136(2):241-7.

8. De Greeff SC, Spanjaard L, Dankert J, Hoebe CJ, Nagelkerke N, de Melker HE. Underreporting of meningococcal disease incidence in the Netherlands: results from a capture-recapture analysis based on three registration sources with correction for false positive diagnoses. Eur J Epidemiol. 2006;21(4):315-21.

9. Trepka MJ, Zhang G, Leguen F. An intervention to improve notifiable diseases reporting using ambulatory clinics. Epidemiol Infect. 2009 Jan;137(1):22-9.

10. Abdool Karim SS, Dilraj A. Reasons for under-reporting of notifiable conditions. S Afr Med J. 1996 Jul;86(7):834-6.

11. Krause G, Ropers G, Stark K. Notifiable disease surveillance and practicing physicians. Emerg Infect Dis. 2005 Mar;11(3):442-5.

12. Pinot de Moira A, Nardone A; ESEN2 group. Varicella zoster virus vaccination policies and surveillance strategies in Europe. Euro Surveill. 2005 Jan;10(1):43-5.
13. Jurković L, Katić M, Ozvacić Z, Stojanović-Spehar S, Vinter-Repalust N. Underestimation of varicella incidence-frequency of requests for medical assistance. Acta Med Croatica. 2003;57(2):117-22. (In Croatian.)

14. Communicable Diseases Act. Official Gazette. 1995 Dec 1;69:5291-300. (In Slovene.)

15. Lopez AS, Guris D, Zimmerman L, Gladden L, Moore T, Haselow DT, et al. One dose of varicella vaccine does not prevent school outbreaks: is it time for a second dose? Pediatrics. 2006 Jun;117(6):e1070-7.

16. Parker AA, Reynolds MA, Leung J, Anderson M, Rey A, Ortega-Sanchez IR, et al. Challenges to implementing second-dose varicella vaccination during an outbreak in the absence of a routine 2-dose vaccination requirement-Maine, 2006. J Infect Dis. 2008 Mar 1;197 Suppl 2:S101-7.

17. Centers for Disease Control and Prevention (CDC). Evaluation of varicella reporting to the National Notifiable Disease Surveillance SystemUnited States, 1972-1997. MMWR Morb Mortal Wkly Rep. 1999 Jan 29;48(3):55-8.

18. Silk BJ, Berkelman RL. A review of strategies for enhancing the completeness of notifiable disease reporting. J Public Health Manag Pract. 2005 May-Jun;11(3):191-200.

19. Perez-Farinos N, Garcia-Gomez L, Ramirez-Fernandez R, Sanz JC, Barranco D, Garcia-Fernandez C, et al. Seroprevalence of antibodies to varicella-zoster virus in Madrid (Spain) in the absence of vaccination. Cent Eur J Public Health. 2008 Mar;16(1):41-4.

20. Fleming DM, Schellevis FG, Falcao I, Alonso TV, Padilla ML. The incidence of chickenpox in the community. Lessons for disease surveillance in sentinel practice networks. Eur J Epidemiol. 2001;17(11):1023-7.

21. Flahault A, Blanchon T, Dorléans Y, Toubiana L, Vibert JF, Valleron AJ. Virtual surveillance of communicable diseases: a 20-year experience in France. Stat Methods Med Res. 2006 Oct;15(5):413-21.

22. Richard JL, Vidondo B, Mäusezahl M. A 5-year comparison of performance of sentinel and mandatory notification surveillance systems for measles in Switzerland. Eur J Epidemiol. 2008;23(1):55-65.

23. Overhage JM, Grannis S, McDonald CJ. A comparison of the completeness and timeliness of automated electronic laboratory reporting and spontaneous reporting of notifiable conditions. Am J Public Health. 2008 Feb;98(2):344-50.

24. Ward M, Brandsema P, van Straten E, Bosman A. Electronic reporting improves timeliness and completeness of infectious disease notification, The Netherlands, 2003. Euro Surveill. 2005 Jan;10(1):27-30.

25. Rolfhamre P, Jansson A, Arneborn M, Ekdahl K. SmiNet-2: Description of an internet-based surveillance system for communicable diseases in Sweden. Euro Surveill. 2006;11(5):103-7.

Received June 16, 2009 Accepted in revised form September 29, 2009 